\title{
Differentially expressed genes implicated in embryo abortion of mango identified by suppression subtractive hybridization
}

\author{
J.H. He ${ }^{1,2}$, F.W. Ma ${ }^{1}$, Y.Y. Chen ${ }^{2}$ and H.R. Shu ${ }^{1,3}$ \\ ${ }^{1}$ College of Horticulture, Northwest A\&F University, Yangling, China \\ ${ }^{2}$ Tropical Crops Genetic Resources Institute, \\ Chinese Academy of Tropical Agricultural Sciences, Danzhou, China \\ ${ }^{3}$ College of Horticulture Science and Engineering, \\ Shandong Agricultural University, Taian, Shandong, China \\ Corresponding author: H.R. Shu \\ E-mail: hrshu@hotmail.com
}

Genet. Mol. Res. 11 (4): 3966-3974 (2012)

Received July 27, 2012

Accepted October 3, 2012

Published November 14, 2012

DOI http://dx.doi.org/10.4238/2012.November.14.4

\begin{abstract}
Embryo abortion in mango severely damages mango production worldwide. The mechanisms by which the mango embryos abort have long been an intriguing question. We used subtractive suppression hybridization to investigate the differentially expressed genes involved in this process. We generated 2 cDNA libraries from normal seed and aborted seed embryos of mango cultivar 'Jinhuang'. One thousand five hundred and seventy-two high-quality expressed sequence tags (ESTs) were obtained, with 1092 from the normal seed tester library and 480 from the aborted seed tester library. These ESTs were assembled into 783 unigenes, including 147 contigs and 636 singletons in contigs; 297 singletons in gene ontology (GO) indicated coverage of a broad range of GO categories. Seven candidate genes from different categories were selected for semi-quantitative PCR
\end{abstract}


analysis, and their possible functions in embryo abortion are discussed. These data provide new insight into the genetic regulation of embryo abortion in mango and may aid in further identification of novel genes and their functions.

Key words: Mango (Mangifera indica L.); Seed embryo abortion; Subtractive suppression hybridization; Expressed sequence tags

\section{INTRODUCTION}

Mango (Mangifera indica L.) is a globally important fruit crop of the tropics and subtropics, particularly in Asia, where it is considered the 'king of fruits' due to its delicate, sweet flavor and nutritional value. However, it has a pronounced tendency to shed fruit at all stages of development until harvest (Singh, 1960). Earlier studies on low fruit set of mango trees suggested that several factors affect fruit drop, including lack of pollination, self-incompatibility and failure of fertilization (Quintana et al., 1984), embryo abortion (Mukherjee, 1953), insect pests (Mohyuddin and Mahmood, 1993), low temperature (Dag et al., 2000), and genotype (Rameshwar and Rao, 1980). Embryo abortion, which hinders normal fruit development, seems to play an important role in induction of the abscission process at early stages of fruit development (Mukherjee, 1953). The aborted mango fruits have a slower growth rate than seeded fruits and normally become malformed, failing to reach full size. This leads to low fruit yields and causes great economic losses in mango-producing areas.

Earlier studies suggested that embryo abortion in mango is due to low or high temperature during flowering (Lakshminarayana and Hernandez-Aguilar, 1975; NúñezElisea and Davenport, 1983). Various problems encountered in pollination and fertilization also lead to embryo abortion in mango fruitlets (Beasley et al., 1999). An anatomical structure assessment of mango fruit revealed that degeneration of the embryo sac leads to embryo abortion (Polito, 1999). Moreover, the effects of embryo abortion on fruit size and quality in different mango cultivars have been well documented (Zora, 2005). So far, these studies and observations have focused on the physiological and anatomical aspects of mango embryo abortion, and the molecular basis of this disorder is unknown. It is necessary to identify the genes that are differentially expressed in aborted tissue to explore the molecular basis of embryo abortion in mango.

We used a PCR-based subtractive hybridization technique to isolate differentially expressed genes in normal and aborted embryo tissues to characterize the molecular causes of embryo abortion. Expression of a few isolated genes was confirmed by RT-PCR. Based on the gene expression profiles, we discuss the probable causes for embryo abortion in mango.

\section{MATERIAL AND METHODS}

\section{Plant material}

Mango cultivar 'Jinhuang' fruits with normal seeds and abortive embryos at stages of fruit-set (20-30 days) were collected from the mango standardized demonstration base of the Tropical Crops Genetic Resources Institute, Chinese Academy of Tropical Agricultural 
Sciences, Hainan, China. All harvested samples were frozen in liquid nitrogen and stored at $-80^{\circ} \mathrm{C}$ prior to use.

\section{Construction of subtracted cDNA libraries by substractive suppression hybridization (SSH)}

Total RNA was isolated from normal or abortive embryo seeds with a plant total RNA extraction kit (Autolab, Beijing, China). Approximately $3 \mu \mathrm{g}$ total RNA from each sample was used to synthesize cDNAs with the BD SMART ${ }^{\mathrm{TM}}$ cDNA Synthesis Kit (Clontech, USA) according to the manufacturer protocol. SSH was performed to generate 2 subtracted cDNA libraries with the PCR-Select ${ }^{\mathrm{TM}}$ cDNA Subtraction Kit (Clontech). For the forward SSH library, normal embryo-derived mRNA was used as a 'tester' and abortive embryo-derived mRNA was used as a 'driver'. A reverse subtracted cDNA pool was made using normal embryo-derived mRNA as a 'driver' and abortive embryo-derived mRNA as a 'tester'. The tester and driver cDNA populations were digested with $R s a$ I to obtain short, blunt-ended fragments. The tester pool was then divided into 2 populations. One population was ligated to adaptor 1 and the other to adaptor 2R. Each tester pool was then hybridized separately with an excess of driver cDNA, and the 2 reactions were mixed together for a second SSH. Two rounds of hybridization and PCR amplification were carried out to normalize and enrich the differentially expressed cDNAs according to the manufacturer protocol. After evaluation of the subtraction efficiency, amplification products generated by the forward and reverse subtracted libraries were cloned into pGEM-T Easy (Promega, USA), transformed into Escherichia coli Top10 competent cells, and plated on selective media. A total of 516 clones from the forward and reverse libraries were randomly picked and cultured for plasmid extraction.

\section{DNA sequencing and sequence analysis}

Nucleotide sequences of the inserted cDNA fragments were determined with the universal M13 sequencing primer and an ABI PRISM ${ }^{\mathrm{TM}} 377$ DNA Sequencer (Applied Biosystems, USA). All sequences were compared to the NCBI (http://www.ncbi.nlm.nih.gov) database with BLASTN/X. Annotated expressed sequence tag (EST) functions were assigned by mapping unigenes on the gene ontology (GO) structure with TIGR Gene Indices Clustering Tools (TGICL).

\section{Semi-quantitative RT-PCR of differentially expressed cDNA}

First-strand cDNAs were synthesized with M-MLV reverse transcriptase (TaKaRa, China) according to the manufacturer protocol. Primers (Table 1) for the candidate genes were designed based on sequences available from 2 SSH libraries. Primers for the internal reference gene Actin were designed based on the sequence of actin from mango (GenBank accession No. HQ830244). RT-PCR conditions were as follows: initial denaturation at $94^{\circ} \mathrm{C}$ for $3 \mathrm{~min}$, followed by 28 cycles of $94^{\circ} \mathrm{C}$ for $30 \mathrm{~s}, 56^{\circ} \mathrm{C}$ for $30 \mathrm{~s}, 72^{\circ} \mathrm{C}$ for $1 \mathrm{~min}$, and a final extension of $5 \mathrm{~min}$ at $72^{\circ} \mathrm{C}$. The experiment was performed in triplicate for each gene, including the notemplate and no-reverse transcriptase controls. 
Table 1. Sequences of primers for semi-quantitative reverse transcription PCR (RT-PCR).

\begin{tabular}{llll}
\hline Clones & Accession No. & \multicolumn{2}{c}{ Primers used for semi-quantitative RT-PCR } \\
\cline { 2 - 3 } & & Forward primer (5'-3') & Reverse primer (5'-3') \\
\hline MaUCE & JK513257 & CGGGGATCTTCCATTTTCTAC & CAGGGCTCCATTGCTCTTTC \\
MaAP2 & JK513603 & GGGCTCTCTCTTTGCTTTCTCT & CCGCTGCCTTATTCCTCTGTA \\
MaHSF & JK513232 & CTGACGAACGAAACTGGAAAA & TGGAGCAAAATGGTGAGACC \\
MaERF & JK513349 & AACGACGAAAGAGCGAAAGG & GAGAATGCACCACCACCATG \\
MaAGA & JK513364 & GCATACGTGGAGATAAAGCCA & CCATAAATCAGCCGAGTAACAGT \\
MaMADS & JK513220 & AGAGATGGGGAGAGGAAAAATT & CGGCTGGAGAAGACAATGAG \\
MaActin & JK513593 & GGGGGTTTCCTGTTCTCTCTT & CACCATGGCTACACCTTTGG \\
\hline
\end{tabular}

\section{RESULTS}

\section{Construction of SSH libraries and overall features of the differential expression profile}

To identify genes differentially expressed in normal seeds (Figure 1A) and abortive embryos of mango cultivar 'Jinhuang' (Figure 1B), 2 subtracted libraries were generated using the SSH technique. After removing low-quality regions and vector and adaptor sequences, the remaining high-quality sequences were clustered and assembled into unigenes to remove redundancy. The average insert of the SSH clones ranged from 200 to $1000 \mathrm{bp}$. Results, including the total number of obtained sequences, contigs, singletons, unigenes, and novelty and redundancy rates, are provided in Table 2 for the forward and reverse subtracted libraries. The nucleotide sequence data of our EST collection have been deposited in the dbEST database at NCBI (http://www.ncbi.nih.gov/dbEST/index.html) with the accession Nos. JK512380JK512855 and JK512856-JK513928.
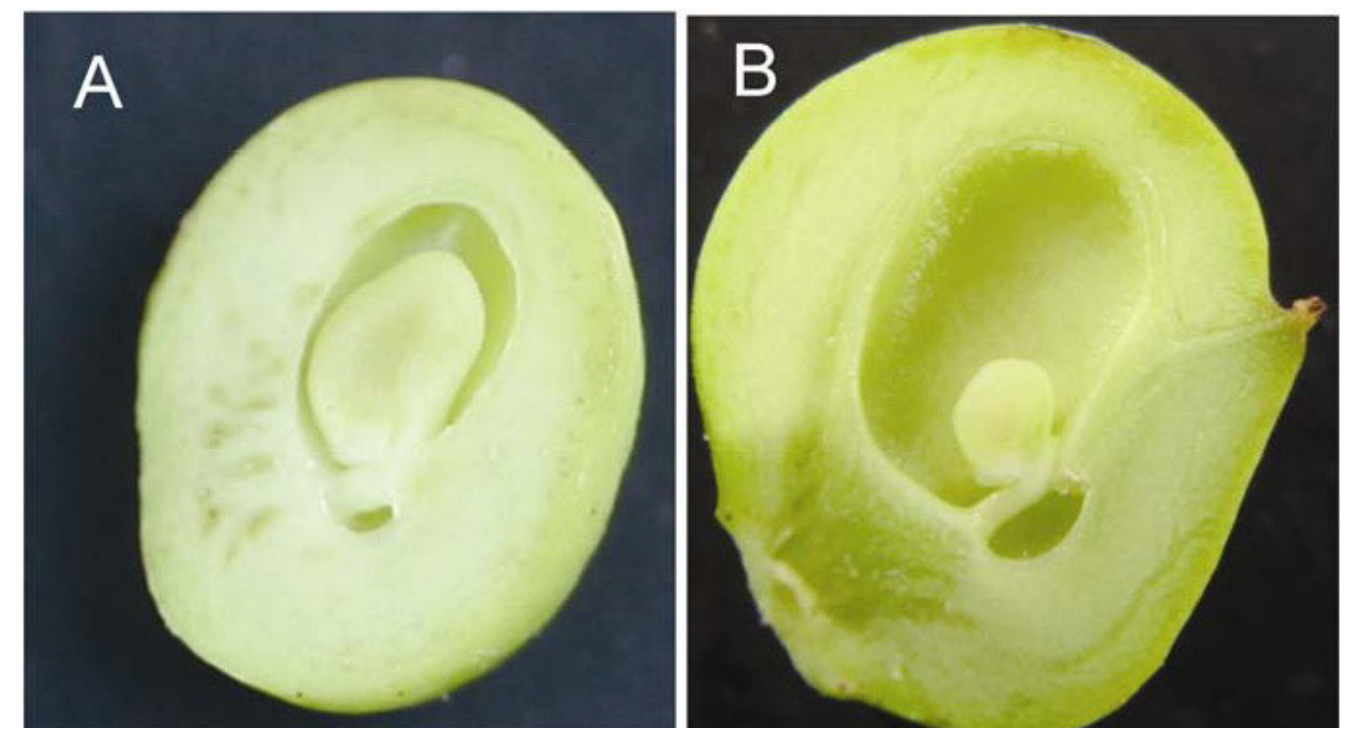

Figure 1. Dissected mango fruit with normal seed (A) and abortive embryo (B) at the mature hard green stage. 
Table 2. General characteristics of the SSH library.

\begin{tabular}{lcccccc}
\hline Library & Assembled ESTs & Contigs & Singletons & Unigenes & Novelty (\%) & Redundancy (\%) \\
\hline Forward & 1073 & 147 & 636 & 783 & 72.97 & 27.03 \\
Reverse & 477 & 44 & 297 & 341 & 71.48 & 28.52 \\
\hline
\end{tabular}

\section{Functional classification of differentially expressed genes}

The unigene set was compared to predicted protein sequences with BLASTX. Annotation of the 783 unigenes from the forward SSH library allowed the functional classification of 252 putative genes. However, $67.8 \%$ (531) of the unigenes had no significant match and could not be classified into GO categories. Unigenes with positive matches to predicted proteins were grouped into 21 categories. The largest proportion of functionally assigned unigenes fell into 5 categories (Figure 2A): Translation, ribosomal structure, and biogenesis (27.78\%); posttranslational modification, protein turnover, and chaperones $(15.48 \%)$; general function prediction only (11.51\%); carbohydrate transport and metabolism $(6.75 \%)$, and energy production and conversion (4.76\%).

In the reverse SSH library of 341 unigenes, 109 were classified into 18 GO categories. However, $68 \%$ (232) of the unigenes did not match known proteins and were considered novel. Among these (Figure 2B), the largest category was posttranslational modification, protein turnover, and chaperones $(22.94 \%)$, followed by the translation, ribosomal structure, and biogenesis category (14.68\%) and the energy production and conversion category $(14.68 \%)$.

\section{Gene expression analysis by RT-PCR}

Based on the data and previous studies on development-related genes in other plant species, 7 candidates were selected to investigate their function in embryo abortion. The selected ESTs showed high homologies with the following genes: AGAMOUS protein (MaAGA, with a similarity of $86 \%$ to GU332504), AP2D domain class transcription factor (MaAP2, with a similarity of $89 \%$ to AY962571), heat shock factor protein (MaHSF, with a similarity of $85 \%$ to HM122592), ethylene response factor (MaEFR, with a similarity of $98 \%$ to EU513281), MADS box protein (MaMADS, with a similarity of $80 \%$ to DQ102369), ubiquitin-conjugating enzyme 28, E2 (MaUCE, with a similarity of $89 \%$ to XM_002284161), and S-adenosylmethionine synthase 1 (MaSAS, with a similarity of $89 \%$ to XM 002512524). To examine the expression patterns of these ESTs in normal and aborted embryo seeds of mango cultivar 'Jinhuang', primers were designed based on the sequencing results and used for semi-quantitative RT-PCR.

The 7 selected ESTs were differentially expressed in the tissues of normal and aborted embryos, indicating they may be involved in embryo abortion and development. These ESTs (MaUCE, MaHSF, and MaSAS) showed similar levels of expression in normal and aborted embryo tissues (Figure 3). However, 4 ESTs (MaAGA, MaAP2, MaERF, and $M a M A D S$ ) were upregulated in normal embryo tissues compared to aborted embryos. It is worth noting that the expression level of MaMADS in aborted embryos is much lower than in normal embryos of 'Jinhuang'. 
A

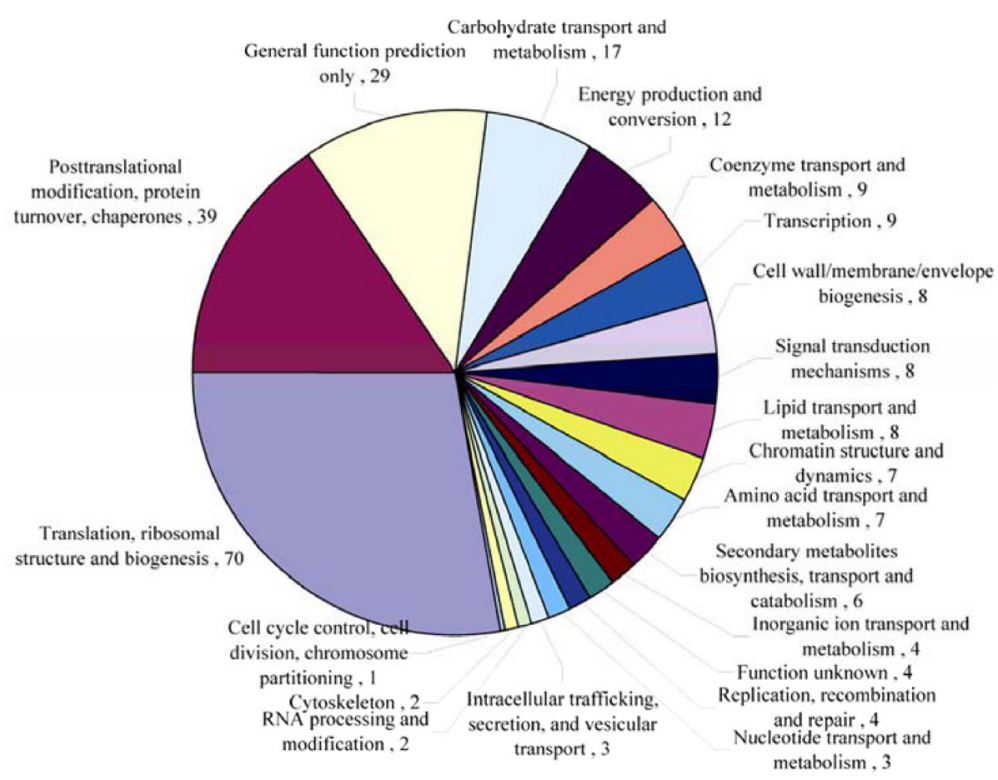

B

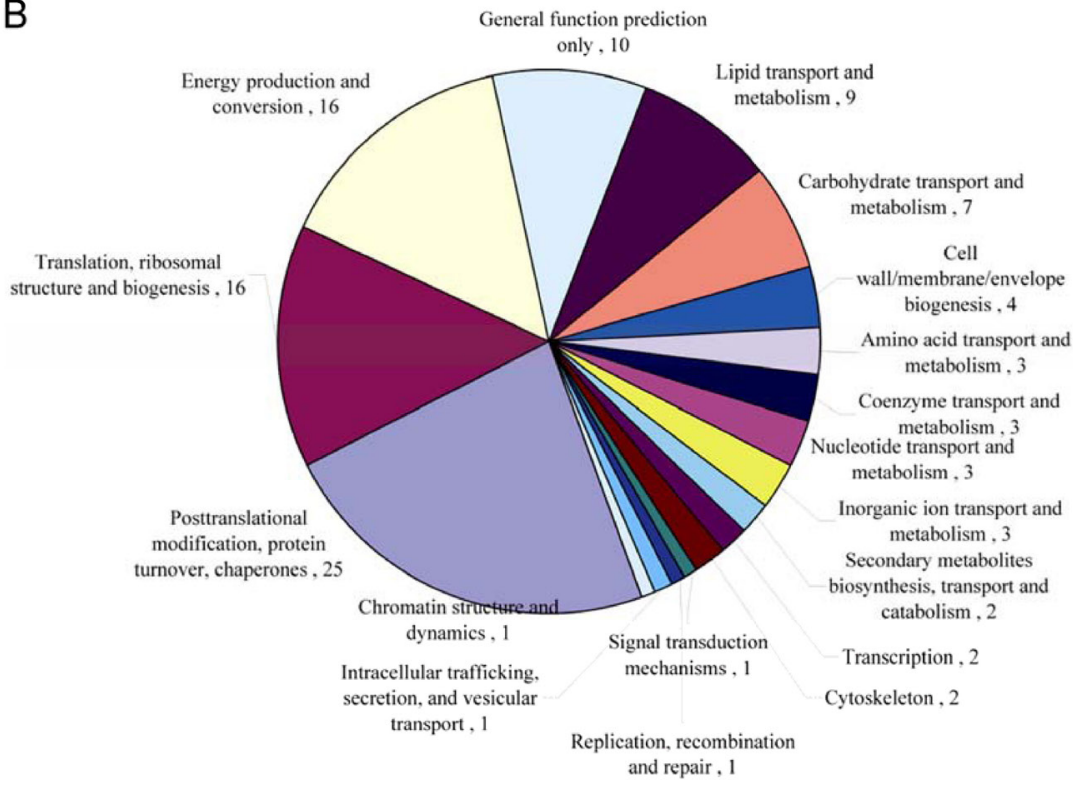

Figure 2. Distribution of all unique sequences annotated for differentially expressed genes into putative functional categories with the respective number of members. Putative functional categories for unique sequences identified for forward libraries (A) and reverse libraries (B). 


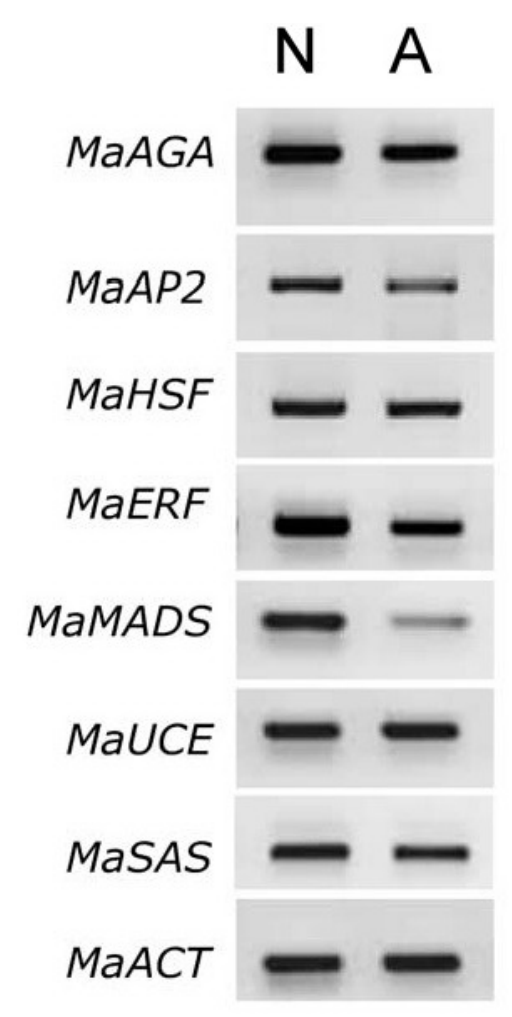

Figure 3. Comparison of the RT-PCR expression patterns of 7 ESTs in the SSH library between fertile and sterile buds. $M a A C T=$ reference gene; $\mathrm{N}=$ normal seeds; $\mathrm{A}=$ abortive embryos.

\section{DISCUSSION}

Although several studies have explored various causes of embryo abortion in mango (Amin, 1967; Subramanyam et al., 1971; Shivashankara and Mathai, 1995), limited information is available on the differentially expressed genes linked to this phenomenon. The cultivar 'Jinhuang' is a leading commercial variety but suffers from severe embryo abortion damage. We used SSH on normal seeds and aborted embryos of mango cultivar 'Jinhuang' to look for genes involved in embryo abortion. Two SSH libraries were constructed to identify genes expressed in normal seeds and in aborted embryos of 'Jinhuang'. We then focused our attention on selected ESTs, which were reported to be associated with embryo development in other plant species, for semi-quantitative analysis to evaluate the relative expression of genes that may be closely related to the molecular mechanism of embryo abortion.

Of the 4 upregulated ESTs, the homologous MADS-box transcription factor MaMADS was upregulated in normal embryo tissues in comparison to aborted embryos (Figure 3). The $M A D S$-box genes encode a large family of transcription factors belonging to the gene clade represented by the Arabidopsis thaliana SEPALLATA3 E function gene (Honma and Goto, 2001). Previous studies have described MADS-box genes as master regulators of flowering 
transition and development of flowers, fruits, and seeds (Ma et al., 2011; Hemming and Trevaskis, 2011). Genome-wide studies suggest that most MADS-box genes are expressed in a variety of organs and cell types at different developmental stages (Rijpkema et al., 2007). In our study, we obtained 1 EST sequence with $86 \%$ homology to the MADS protein. Sequence and phylogenetic analyses revealed that the MaMADS box transcription factor belongs to the MEF2 (myocyte enhancer factor 2)-like/Type II subfamily of MADS. Loss-of-function mutations in multiple $M A D S$-box genes can convert floral organs to leaf-like structures, and ectopic expression of $M A D S$-box genes can convert leaves into floral organs, illustrating the critical role $M A D S$-box genes play during floral development (Trevaskis et al., 2007). The MaMADS box gene was highly upregulated in normal embryos but downregulated in aborted embryos, further confirming that the $M A D S$ transcription factor may act in a dominant-positive manner in embryo development and regulatory pathways. Our results therefore suggest that further studies on this gene will provide new insights into the mechanism of embryo abortion.

Ubiquitin-conjugating enzyme E2 (UCE) constitutes a family of conserved proteins that play a key role in ubiquitin-dependent protein degradation in eukaryotes. Functional analyses of potential ubiquitin-conjugating enzymes have been well-documented in mammalian cells. Evidence suggests that ubiquitin-conjugating E2 enzyme is essential for male fertility in mouse (Roest et al., 1996), and provirus integration into a gene encoding a ubiquitin-conjugating enzyme results in a placental defect and embryonic lethality (Harbers et al., 1996). However, its function in parthenogenesis has not been determined. The gene encoding S-adenosylmethionine synthase 1 (SAS) participates in embryo development and may be a key factor for the initiation of embryo abortion (Xiang et al., 2007). Other studies have indicated that the gene encoding heat shock factor protein $(H S F P)$ may be involved in embryo development (Podrabsky and Somero, 2007). However, the potential functions of these genes in mango embryo abortion have not been determined. In our study, we noted no significant changes in expression of MaUCE, MaHSF, and MaSAS in normal and aborted embryos. We imagine that a very small proportion of these genes contribute specifically to mango embryo development. Further experiments are required to test this speculation.

We also note that some of ESTs obtained from 2 SSH libraries are novel genes and no annotations are available to their functions associated with embryo development/abortion. These sequences, therefore, might consist of unidentified or novel genes in embryo development, and their association with embryo abortion must be clarified. The initial goal of this study was to identify genes that control or are associated with embryo abortion in normal and abortive mango embryos. Nevertheless, the preliminary work involved only EST isolation and only a few genes related to embryo abortion were identified. In view of this problem, further research is required to characterize genes that may be associated with embryo abortion, in particular, the related control genes or transcriptional factors. Because of the limited number of identified genes, we still need to screen whole libraries to identify the functions of these differentially expressed genes in embryo development/abortion.

This study provides fundamental information on the genetic basis of the development of mango. Although the results of this study are based on limited clones and thus cannot be considered fully representative, it is clear that many transcriptionally regulated genes differentially accumulate in 2 types of mango embryo. The MaMADS gene may play an important role in embryo development. Overall, these molecular and bioinformatic data provide basic EST sequences and a step forward in our understanding of gene function and regulatory pathways linked to embryo abortion in mango. 


\section{ACKNOWLEDGMENTS}

Research supported by the Special Fund for Agro-Scientific Research in the Public Interest for "Technical study and demonstration of mango industry" (Grant \#201203092).

\section{REFERENCES}

Amin HD (1967). Development of white corky tissue in a mango fruit (Mangifera indica L.). Navsari Agric. Coll. Mag. 6: 14-17.

Beasley DR, Joyce DC and Hofman PJ (1999). Effect of preharvest bagging and of embryo abortion on calcium levels in "Kensington Pride" mango fruit. Aust. J. Exp. Agric. 39: 345-349.

Dag A, Eisenstein D and Gazit S (2000). Effect of temperature regime on pollen and the effective pollination of "Kent" mango in Israel. Sci. Hortic. 86: 1-11.

Harbers K, Muller U, Grams A, Li E, et al. (1996). Provirus integration into a gene encoding a ubiquitin-conjugating enzyme results in a placental defect and embryonic lethality. Proc. Natl. Acad. Sci. U. S. A. 93: 12412-12417.

Hemming MN and Trevaskis B (2011). Make hay when the sun shines: the role of MADS-box genes in temperaturedependant seasonal flowering responses. Plant Sci. 180: 447-453.

Honma T and Goto K (2001). Complexes of MADS-box proteins are sufficient to convert leaves into floral organs. Nature 409: 525-529.

Lakshminarayana S and Hernandez-Aguilar P (1975). Effect of orchard heating in reducing parthenocarpic fruits in "Haden" mango. Proc. Fla. State Hortic. Soc. 88: 502-505.

Ma C, Wang Y, Wang Y, Wang L, et al. (2011). Identification of a sugar beet BvM14-MADS box gene through differential gene expression analysis of monosomic addition line M14. J. Plant Physiol. 168: 1980-1986.

Mohyuddin AI and Mahmood R (1993). Integrated control of mango pests in Pakistan. Acta Hortic. 341: 467-483.

Mukherjee SK (1953). The mango: its botany, cultivation, uses and future improvement, especially as observed in India. Econ. Bot. 7: 130-162.

Núñez-Elisea R and Davenport TL (1983). Abscission and ethylene production of mango (Mangifera indica L.) fruit cv Tommy Atkins. Proc. Fla. State Hort. Soc. 96: 185-188.

Podrabsky JE and Somero GN (2007). An inducible 70 kDa-class heat shock protein is constitutively expressed during early development and diapause in the annual killifish Austrofundulus limnaeus. Cell Stress Chaperones 12: 199-204.

Polito VS (1999). Seedlessness and parthenocarpy in Pistacia vera L. (Anacardiaceae): temporal changes in patterns of vascular transport to ovules. Ann. Bot. 83: 363-368.

Quintana EG, Nanthaghai P, Hiranpradit DB, Mendoza J, et al. (1984). Growth and Development of Mango. In: Mango: Fruit Development, Postharvest Physiology and Marketing in ASEAN (Mendoza DB and Wills RBH, eds.). ASEAN Food Handling Bureau, Kuala Lumpur, 21-39.

Rameshwar A and Rao SN (1980). Why fruit drop in mango? Intensive Agricult. 18: 17-18.

Rijpkema AS, Gerats T and Vandenbussche M (2007). Evolutionary complexity of MADS complexes. Curr. Opin. Plant Biol. 10: 32-38.

Roest HP, van Klaveren J, de Wit J, van Gurp CG, et al. (1996). Inactivation of the HR6B ubiquitin-conjugating DNA repair enzyme in mice causes male sterility associated with chromatin modification. Cell 86: 799-810.

Shivashankara KS and Mathai CK (1995). Physiological diversity among the potentially productive branches of regular and irregular bearing mango cultivars. Photosynthetica 31: 135-140.

Singh LB (1960). The Mango: Botany, Cultivation and Utilization. Leonard Hill, London.

Subramanyan H, Krishnamurthy S, Subhadra NV, Dalal VB, et al. (1971). Studies on internal breakdown, a physiological ripening disorder in 'Alphonso' mangoes (Mangifera indica L.). Trop. Sci. 13: 203-210.

Trevaskis B, Tadege M, Hemming MN, Peacock WJ, et al. (2007). Short vegetative phase-like MADS-box genes inhibit floral meristem identity in barley. Plant Physiol. 143: 225-235.

Xiang X, Zhang YS, Huang SZ, Ou L, et al. (2007). Application of suppressive subtraction hybridization in the study of embryo abortion related to gene expression in litchi (Litchi chinensis Sonn.). Acta Hort. 763: 83-90.

Zora S (2005). Embryo abortion in relation to fruit size, quality, and concentrations of nutrients in skin and pulp of mango. J. Plant Nutr. 28: 1723-1737. 